\title{
Precipitação Geoquímica em Ambientes Evaporíticos/Hipersalinos - o caso das Salinas Solares do Brasil
}

\author{
Geochemical precipitation in evaporitic/hypersaline environments - the case of coastal solar \\ saltworks of Brazil.
}

\author{
COSTA, D. F. S.; SILVA, D. E. M.; SOUZA, A. C. D.; SALDANHA, D. S.; BATISTA, A. I. L. \\ diogenesgeo@gmail.com;
}

\begin{abstract}
Resumo
As salinas têm sido utilizadas pelo homem há milênios, sendo compostas por uma série de tanques rasos e interconectados, nos quais a água do mar/estuário é captada e transferida de um tanque para outro por gravidade ou por bombeamento, onde a produção do sal se dá a partir da saturação e precipitação dos sais por evaporação solar. Objetivou-se identificar e descrever o processo de deposição e formação de sais em ambientes evaporíticos artificiais (salinas solares) do Brasil. Verificou-se que a precipitação de sais nas salinas incluiu os compostos menos solúveis na base para o mais solúvel na parte superior da sequência, na seguinte ordem: carbonatos $\left(\mathrm{CaCO}_{3}\right)$, gipsita $\left(\mathrm{CaSO}_{4} 2 \mathrm{H}_{2} \mathrm{O}\right)$, halita $(\mathrm{NaCl})$, sais de potássio - silvinita (sistema $\mathrm{NaCl}-\mathrm{KCl}$ ), e magnésio - biscofita $\left(\mathrm{MgCl}_{2} \cdot 6 \mathrm{H}_{2} \mathrm{O}\right)$. Os carbonatos precipitam-se em salinidades em torno de 50 a $80 \mathrm{gL}^{-1}$, seguidos dos sulfatos, que se precipitam gradativamente ao longo dos evaporadores da salina, até atingir seu máximo de precipitação entre 150 e $160 \mathrm{gL}^{-1}$. As maiores quantidades de cristais de halita são formadas quando a concentração total da salinidade atingiu um valor acima de $240 \mathrm{gL}^{-1}$. A variação e sequência de deposição evaporítica nas salinas apresentam limites diferentes de dissolução no ambiente, vindo a precipitar-se quando atingem saturações máximas.
\end{abstract}

Palavras-chave: Produção de Sal, Áreas úmidas, Rio Grande do Norte.

\begin{abstract}
Solar saltworks have been regulated by human activity for millennia, and they comprise a series of interconnected shallow hypersaline ponds, in which the seawater is pumped or fed by gravity to the next pond, where salt production it occour from saturation and precipitation of the salts by solar evaporation. This research had as main objective to describe the deposition and precipitation process of salts in artificial evaporitics environments (solar saltworks) in Brazil. It verified that the precipitation of salts in this environments included the less from more solubility salts, at the follow order: carbonates $\left(\mathrm{CaCO}_{3}\right)$, gypsum $\left(\mathrm{CaSO}_{4} 2 \mathrm{H}_{2} \mathrm{O}\right)$, halite $(\mathrm{NaCl})$, potassium salts - sylvinite $(\mathrm{NaCl}-\mathrm{KCl}$ system), and magnesium salts - bischofite $\left(\mathrm{MgCl}_{2} \cdot 6 \mathrm{H}_{2} \mathrm{O}\right)$. The carbonates precipitate between salinities at 50 to $80 \mathrm{~g} . \mathrm{L}^{-1}$, followed to sulphates, that que precipitate gradually around evaporators' ponds, until reach the max between 150 e 160 g.L ${ }^{-1}$. The more quantities of the halite crystals are formad when total salinity concentration reaches above $240 \mathrm{gL}^{-1}$. The variation and sequence of the evaporitc deposition in solar saltworks is clearly explained by concept of fracioned precipitation of salts from seawater. It verified that the different salts showed different limits of the dissolution in the environment, come precipitating when reach max saturation.
\end{abstract}

Keywords: Salt Production, Wetlands, Rio Grande do Norte.

\section{INTRODUÇÃO}

Alguns dos primeiros estudos sobre os ambientes evaporíticos/hipersalinos se deram na Costa Trucial do Golfo da Pérsia (CURTIS et al., 1963), atual território dos Emirados Árabes 
Unidos. Esses ambientes geralmente são encontrados em planícies de supramaré, sendo essencialmente de baixo aporte de sedimentos, altas taxas de evaporação e formação natural de cristais de sais (SILVA et al., 2000). Em resumo, o evaporito constitui-se em uma rocha sedimentar que se forma por precipitação dos sais contidos em uma solução química inorgânica, em função da evaporação em ambiente salino (salmouras de lagos e mares interiores que se evaporam) (KENDALL, 1984).

A formação de evaporitos marinhos requer condições de extrema restrição de circulação de águas marinhas aliada a condições climáticas específicas, onde possibilitem que a razão de evaporação seja superior à taxa de influxo marinho. Tal restrição à livre circulação de águas marinhas, ou barreiras, pode-se ocorrer em função de alterações nas condições geomorfológicas, estruturais, orgânicas (recifes) ou artificiais (construção de salinas solares). Tais ambientes estão propensos a inundações periódicas e deposição evaporítica, dominada por carbonatos, sulfatos e halitas, também podendo ocorrer em zonas interiores, porém são mais comuns em áreas costeiras de topografia plana em regiões áridas, desenvolvendo-se em resposta a duas condições ambientais (e.g. deflação de sedimentos de superfície, acumulação, saturação e precipitação de sais (EVANS, 1970; AL FARRAJ, 2005; OREN, 2009).

Desde a publicação de Curtis et al. (1963), a Costa Trucial, a Península de Qatar e a Baja Califórnia, tornaram-se áreas clássicas para o estudo da formação de evaporítos em planícies hipersalinas. Essas zonas, tornaram-se de relevante interesse econômico em virtude dessa associação de fácies sedimentares tais como esteiras algálicas formadas na região de intermaré, nódulos e estruturas enterolíticas de sulfato de cálcio $\left(\mathrm{CaSO}_{4}\right)$, tanto gipsita como anidrita, característico da porção supramaré, e cubos bem como formas esqueletais de halita em uma matriz carbonática ou siliciclástica (SILVA et al., 2000), além de serem importantes para o início da formação do petróleo (AL FARRAJ, 2005).

No caso específico dos ambientes evaporíticos artificiais, as salinas solares são os principais exemplos. As salinas solares são encontradas em regiões tropicais e subtropicais em todo o mundo, nas quais a água do mar é evaporada naturalmente em uma série de lagos artificiais rasos, hipersalinos e interligados para a produção comercial de halita $(\mathrm{NaCl})$ e por vezes outros sais (DAVIS, 2000; OREN, 2009). Estes sistemas costeiros representam habitats antropogênicos típicos das zonas de supramaré e são utilizados pelo homem a milênios para a produção de sal (LÓPEZ et al., 2010). As primeiras referências de que se dispõe sobre a extração de sal da água do mar são oriundas da China, durante a dinastia do imperador Huang, até o ano 2.500 a.C. O procedimento usado era basicamente o mesmo que se aplica atualmente em algumas localidades da África, América do Sul e Oceania, e consistia em represar a água do mar em diques de argila, aguardando a 
precipitação do sal marinho, obtendo o predomínio de $\mathrm{NaCl}$ e grande conteúdo em sais de cálcio, magnésio, etc. (BAAS-BECKING, 1931).

Considerando-se a incipiente produção de material científico sobre as salinas solares brasileiras, este artigo de revisão traz uma descrição pormenorizada da precipitação geoquímica dos sais ao longo do circuito de produção de sal em salinas solares. A partir dos referenciais teóricos, espera-se que este trabalho venha a contribuir com um maior conhecimento sobre a dinâmica geoquímica em ambientes evaporíticos artificiais, servindo como base de consulta para futuras investigações científicas analíticas.

\section{SALINAS BRASILEIRAS}

As salinas solares têm sido estudadas extensivamente ao longo do globo a nível de dinâmica dos processos ecológicos e características hidrogeoquímicas, principalmente nos Estados Unidos, Austrália, Itália, China, Tunísia, Grécia, Espanha, Israel, México e Índia (TAKEKAWA et al., 2006; COLEMAN, 2009; GIORDANO; BEARDALL, 2009; HUAI-KE; GUAN-QIANG, 2009; KHEMAKHEM et al., 2010; KOROVESSIS; LEKKAS, 2006; LÓPEZ et al., 2010; OREN et al., 2009; ORTIZ-MILAN; DAVIS, 2009; RAHAMAN et al., 2009). Todavia, encontra-se pouca informação científica sobre as salinas brasileiras, onde sequer os processos hidrogeoquímicos das salmouras foram suficientemente caracterizados (SOUTO et al., 2000; COSTA, 2013).

No Brasil, ambientes evaporíticos/hipersalinos foram descritos apenas no litoral dos estados de Sergipe e Rio de Janeiro (SILVA et al., 2000), porém ocorrem em grandes áreas dos estados do Rio Grande do Norte e Ceará, conforme atestam algumas pesquisas (e.g. MOREIRA et al., 1989; MELO et al., 2008; MENDES et al., 2008) sobre solos hipersalinos (Gleissolo sálico Brasil/Solonchaks - FAO) nestes estados. Estas áreas assumiram especial importância desde a colonização do Brasil, sendo ocupadas para construção de salinas solares (VITA et al., 2007; COSTA et al., 2013), principalmente em virtude das características específicas desse tipo de solo, como alta salinidade e impermeabilidade (KENDALL; HARWOOD, 1996; GUO et al., 2006). Estes solos alcalinos $(\mathrm{pH}>8,0)$ apresentam horizonte sálico $\left(1: 1\right.$ em água), com $\left(\mathrm{Cl}^{-}\right)>\left(\mathrm{SO}_{4}{ }^{2-}\right)>$ $\left(\mathrm{HCO}_{3}{ }^{-}\right)$, caracterizado por uma saturação de sódio $\left(100 \mathrm{Na}^{+} / \mathrm{CTC}\right)$ maior ou igual a $15 \%$ (CEC no extrato de saturação maior ou igual a $7 \mathrm{dS} / \mathrm{m}$, a $25^{\circ} \mathrm{C}$ ) em alguma época do ano, sendo largamente limitados às zonas de clima árido e semiárido em regiões costeiras (EMBRAPA, 1999; FAO, 2006).

No Brasil, as regiões em que tradicionalmente se desenvolve a exploração do sal marinho se encontram em zonas com forte insolação diária, nas proximidades de estuários, com predomínio de solos argilosos que favorecem a impermeabilização do terreno. Os índices pluviométricos das 
referidas áreas costumam ser baixos ou, pelo menos com um período de chuvas concentrado em poucos meses do ano. Outra característica climatológica importante é a presença de fortes ventos que permitam a aceleração da evaporação da água (COSTA et al., 2010; COSTA, 2013).

Todavia, ao longo da linha de costa brasileira, apenas no litoral setentrional do estado do Rio Grande do Norte estão situadas as maiores empresas salineiras do país, principalmente nas margens dos seus estuários (Fig. 01). Essas salinas são responsáveis por 97\% do sal marinho produzido e exportado no país, influenciando diretamente nas economias locais e regionais através a geração de empregos e pagamento de impostos. Estas salinas constituem ecossistemas artificiais explorados para a extração de sal marinho, sendo compostos por uma série de tanques rasos $(20-200 \mathrm{~cm})$ e interconectados, nos quais a água do mar/estuário é captada e transferida de um tanque para outro por gravidade ou por bombeamento. Ao longo desse circuito, esta água vai evaporando gradativamente, o que aumenta a saturação de sais até se atingir uma salmoura com saturação de $240 \mathrm{gL}^{-1}$ de sais, já no estágio final de cristalização do cloreto de sódio (COSTA, 2013).

A localização das salinas ao longo dos estuários nesse litoral, deve-se, à conjuntura de fatores ambientais como clima semiárido, com altas temperaturas $\left(>28^{\circ} \mathrm{C}\right)$, baixa precipitação pluviométrica $\left(<800 \mathrm{~mm} \cdot \mathrm{ano}^{-1}\right)$ e altas taxas de evaporação, juntamente com a disponibilidade de água do estuário e extensas planícies hipersalinas. Devido aos fatores climáticos, durante boa parte do ano (período de estiagem) os estuários se tornam negativos/hipersalinos (MARGALEF, 1974; MIRANDA et al., 2004), atingindo valores de salinidade acima de $40 \mathrm{gL}^{-1}$ (SILVA et al., 2009), favorecendo assim as salinas com a captação de uma água já com elevada saturação de sais (COSTA et al., 2010).

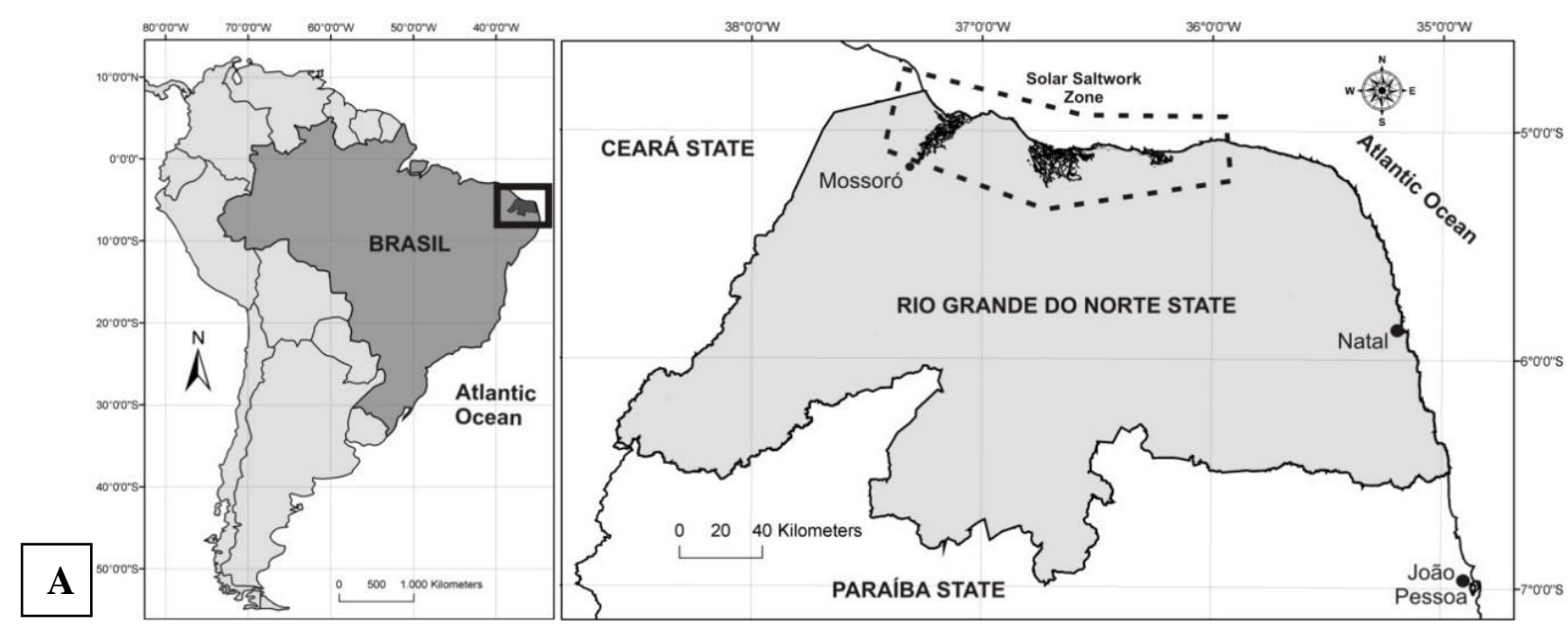




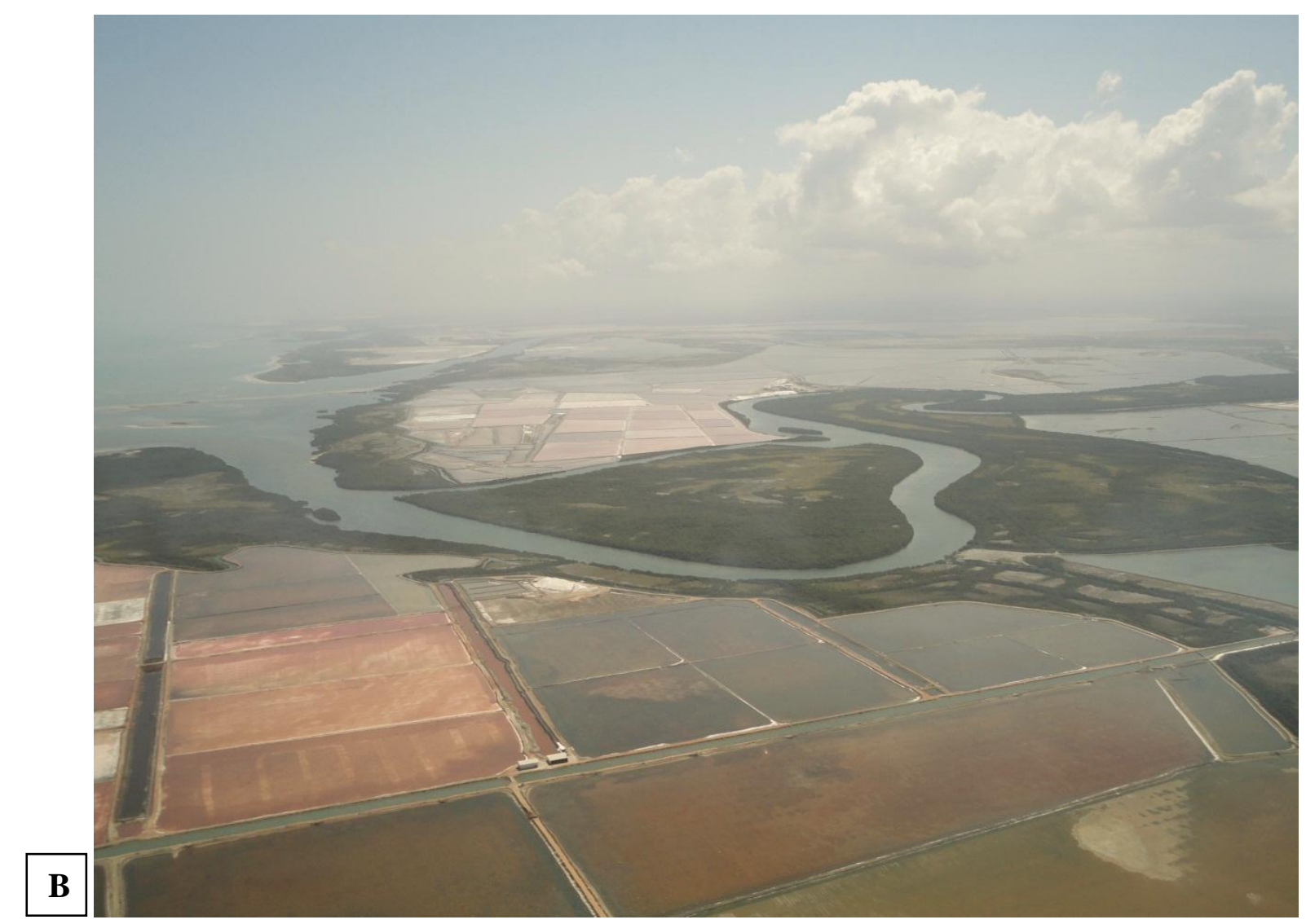

Figura 01. Localização da zona salineira no litoral semiárido do Brasil (A - Mapa de localização / B Fotografia ilustrativa).

Em termos de manejo, a água do mar é bombeada para os primeiros conjuntos de tanques evaporadores, até evaporar a uma salinidade definida, sendo então transferida para a próxima série de evaporadores, enquanto aumenta a salinidade em cada etapa. Finalmente a salmoura saturada com $\mathrm{NaCl}(99,7 \%)$ é transferida para os tanques cristalizadores (profundidade $<20 \mathrm{~cm}$ ), nos quais se tem a precipitação final dos sais de halita. Portanto, as salinas consistem de um gradiente contínuo de salinidade, em que a concentração de sais em cada evaporador é mantida aproximadamente constante ao longo de todo o ano (COSTA, 2013). Esse fluxo da água ao longo dos vários tanques cria um gradiente com características físico-químicas estáveis, principalmente com relação à salinidade (BAUDIN, 1980; BRITTON; JOHNSON, 1987; JAVOR, 1989; PEDRÓS-ALIÓ et al., 2000; OREN, 2002). A organização espacial dos tanques nas salinas e suas diferentes profundidades, necessários para o processo de produção do sal, favorecem o desenvolvimento de um alto grau de heterogeneidade espacial, com vários microambientes (EVAGELOPOULOS et al., 2008; DAVIS, 2000; LÓPEZ et al., 2010).

A qualidade e a produtividade da extração de sal de algumas salinas melhoraram ao longo das duas últimas décadas, mediante novos sistemas (e.g. modernização da construção, biotecnologia, disposição dos tanques por causa do aumento da taxa de evaporação), com a 
finalidade de facilitar a ação dos processos físicos que podem provocar um aumento na produtividade (COSTA et al., 2010, 2013).

\section{PRECIPITAÇÃO/DEPOSIÇÃO GEOQUÍMICA}

A análise da dinâmica geoquímica está fortemente associada ao conceito da precipitação fracionada dos sais da água do mar (Fig. 02), proposto por Copeland (1967) e Baseggio (1973), de acordo com a solubilidade dos vários elementos e moléculas. Assim, a precipitação de sais inclui os compostos menos solúveis na base, até o mais solúvel na parte superior da sequência. Verifica-se a relação direta entre a elevação da saturação de sais na salmoura, onde os diferentes sais formados apresentam limites diferentes de dissolução no ambiente, vindo a precipitar-se quando atingem saturações máximas (OREN, 2002).

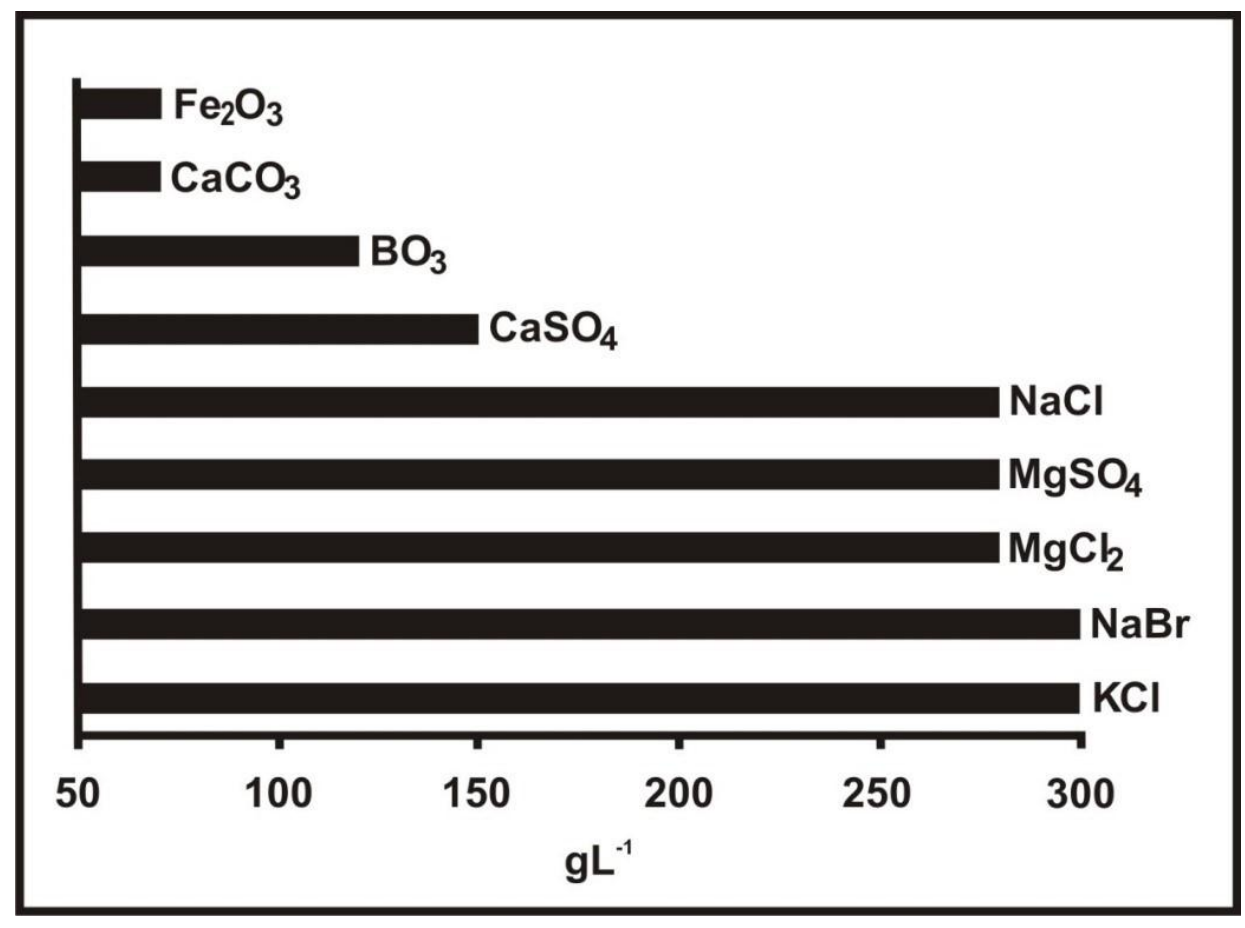

Figura 02. Precipitação dos sais segundo Copeland (1967) e Basegio (1973). Fonte: COSTA (2013).

Em uma fase inicial, o cálcio primeiramente se associa aos carbonatos, formando a calcita $\left(\mathrm{CaCO}_{3}\right)$, precipitando-se já em salinidades em torno de 50 a 80 g.L.- Em seguida, este elemento passa a se associar aos sulfatos, formando tanto a gipsita $\left(\mathrm{CaSO}_{4} 2 \mathrm{H}_{2} \mathrm{O}\right)$ quanto a anidrita $\left(\mathrm{CaSO}_{4}\right)$, porém ao absorver umidade transforma-se em gipsita, com aumento de volume). Estes sais precipitando-se gradativamente e em grandes quantidades ao longo dos evaporadores da salina, até atingir seu máximo de precipitação entre 150 e 160 g.L $\mathrm{L}^{-1}$, formando em algumas salinas uma crosta com vários centímetros de espessura (Fig. 03). Este processo representa as duas primeiras fases de 
evaporação, onde os depósitos desse tipo de gesso são caracteristicamente encontrados no substrato das salinas entre este intervalor de salinidade (OREN, 2002; 2009).

Por sua vez, cristais de halita $(\mathrm{NaCl})$ se formam a partir da sua precipitação mais intensa quando a saturação salina atinge valores superiores a 210 g.L ${ }^{-1}$ (Fig. 04) (OREN, 2002). Os sais mais solúveis como a silvita (sistema $\mathrm{NaCl}-\mathrm{KCl})$, a epsomita $\left(\mathrm{MgSO}_{4} 7 \mathrm{H}_{2} \mathrm{O}\right)$, a biscofita $\left(\mathrm{MgCl}_{2} \cdot 6 \mathrm{H}_{2} \mathrm{O}\right)$, a carnalita $\left(\mathrm{KCl}_{\mathrm{MgCl}} \cdot 6 \mathrm{H}_{2} \mathrm{O}\right)$ e a polialita $\left(\mathrm{K}_{2} \mathrm{SO}_{4} \cdot \mathrm{MgSO} \cdot 2 \mathrm{CaSO}_{4} \cdot 2 \mathrm{H}_{2} \mathrm{O}\right)$, associam-se à halita em alguns depósitos com saturações de sais superiores a 280 g.L ${ }^{-1}$, constituindo-se em importantes fontes de potássio e magnésio. Também é considerada a presença de outros compostos, de acordo com variações físicas e químicas da solução salina durante as várias fases de evaporação (BASEGGIO, 1973; KENDALL; HARWOOD, 1996).

Todavia, após a maior parte do $\mathrm{NaCl}$ precipitar-se para o substrato das salinas (entre 240 e 270 g.L $\mathrm{L}^{-1}$ ), ainda permanecem salmouras concentradas ("água-mãe” ou "bitterns" em inglês), contendo principalmente concentrações elevadas de magnésio $\left(\mathrm{Mg}^{2+}\right)$, potássio $\left(\mathrm{K}^{+}\right)$, cloreto $\left(\mathrm{Cl}^{-}\right)$e sulfato $\left(\mathrm{SO}_{4}{ }^{2-}\right)(\mathrm{OREN}, 2002)$. Estas salmouras são geralmente descartadas no mar ou para processamento adicional, para a colheita de potássio ou outros sais (JAVOR, 1989; DAVIS, 2000; 2002; OREN 2003).

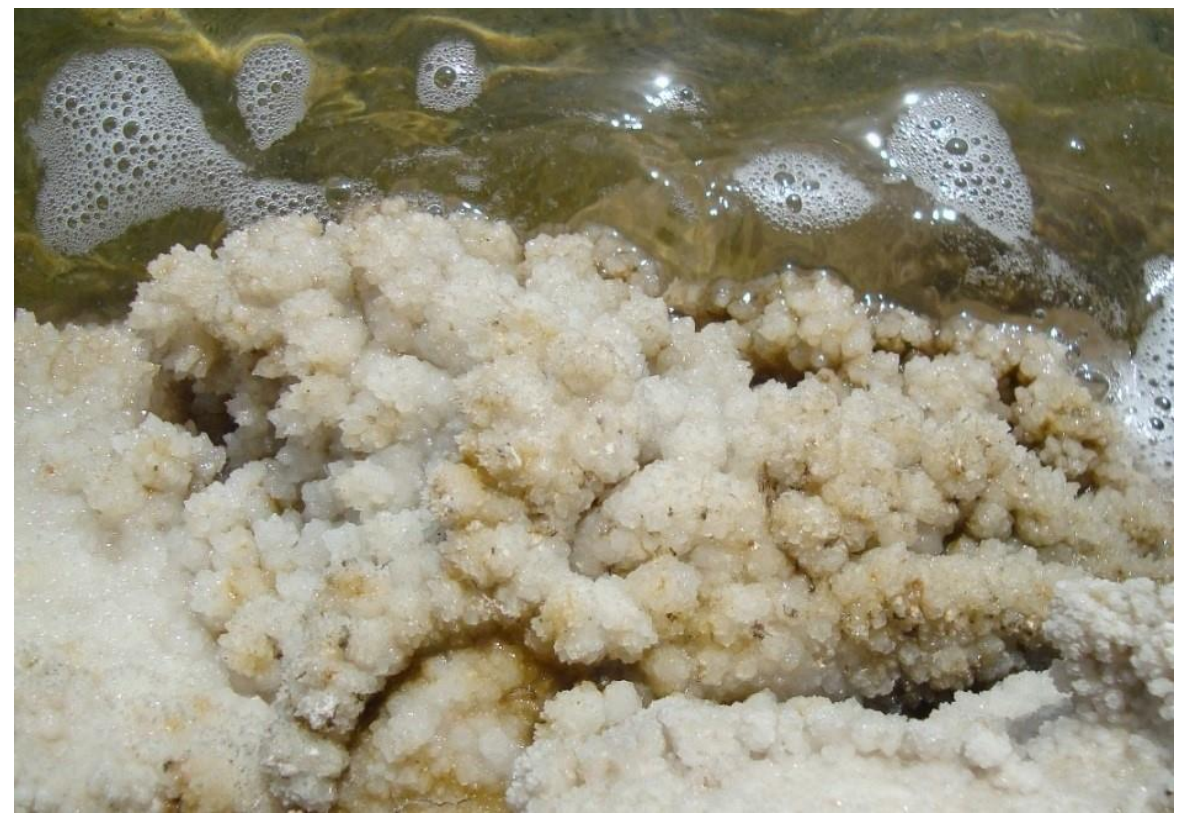

Figura 03. Cristais de gipsita $\left(\mathrm{CaSO}_{4} 2 \mathrm{H}_{2} \mathrm{O}\right)$ formados nos evaporadores com salinidades de 150 e 160 g. $\mathrm{L}^{-1}$. 


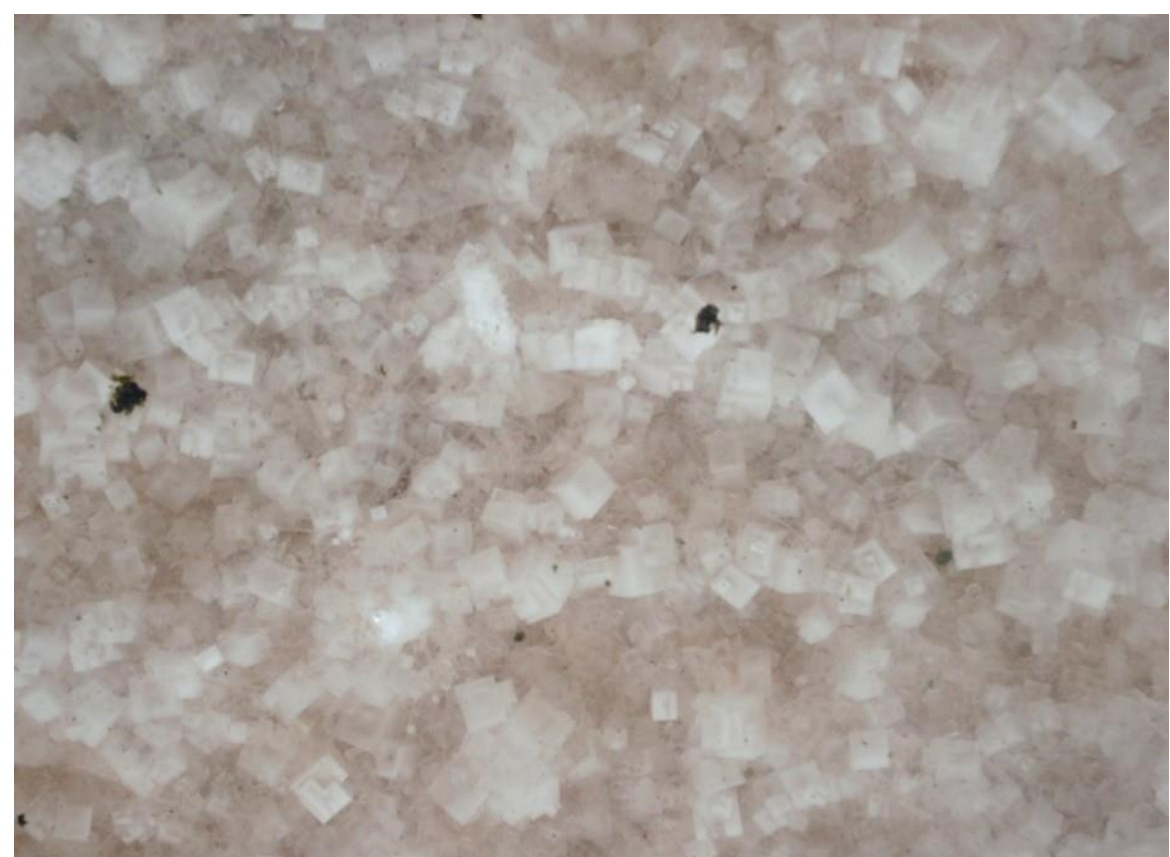

Figura 04. Tanque cristalizador onde se deu a precipitação final dos cristais de halita $(\mathrm{NaCl})$ a partir de 210 g...- ${ }^{-1}$.

Em resumo, quando todo o carbonato de cálcio, sulfato de cálcio, e $83 \%$ da halita é cristalizada a partir d'água do mar por evaporação solar e manejo da salmoura, a "água-mãe” é com poucas exceções colocada de volta para o mar. Em alguns casos, como na Espanha, são utilizadas para a produção de alguns sais, como a epsomita, biscofita e bromo, mas não para a produção de sais de potássio. Porém, no prosseguimento da evaporação, uma mistura complexa de halita, silvita, além de sais duplos de potássio, sódio e magnésio começam a cristalizar, onde a recuperação de produtos comercializáveis torna-se difícil e ineficiente. No entanto, na ausência ou quase ausência de sulfato, a "água-mãe" pode ser facilmente processada para recuperar a alta pureza da silvita, epsomita, biscofita e hexa-hidrato de cloreto de magnésio com excelente eficiência por uma combinação de evaporação solar e cristalização fracionada (FERNÂNDEZ-LOZANO, 1973).

Já o magnésio é profundamente presente na salmoura de evaporitos, assim como o cloreto $(9,44 \%)$, sulfato $(6,5 \%)$ e brometo $(0,22 \%)$. De maneira associada, o cloreto de magnésio ocorre na natureza como biscofita e como carnalita, ambos de origem oceânica. Todavia, é importante ressaltar que a matéria-prima da indústria de magnésio é, no entanto, o hidróxido de magnésio, sendo processado a base de ácido clorídrico para se obter o cloreto de magnésio. O valor potencial desse produto resultante como matéria-prima é representativo, mas envolve a separação de sais diferentes para se obter o cloreto de magnésio em uma forma relativamente pura (JADHAV, 1983). A produção de hexa-hidrato de cloreto de magnésio cristalino por evaporação solar da "água mãe" poderia gerar um produto adequado para a produção electrolítica de metais de magnésio. Porém, 
esse processo se torna praticamente inviável, principalmente devido ao elevado valor do teor de sulfato (DERBY, 1916; JADHAV, op. cit.).

\section{CONSIDERAÇÕES FINAIS}

A partir desta análise, verificou-se que as salinas são sistemas integrados do ponto de vista geoquímico, sendo compostas por um mosaico de habitats cuja salinidade é um dos principais agentes reguladores da dinâmica de precipitação dos diferentes sais encontrados em cada habitat. Por sua vez, é exatamente a logística da produção de sal marinho que implica nessa segmentação espacial de habitas, que apresentam como resposta imediata esse gradiente espacial da salinidade, até o setor final de produção (cristalizadores). A discussão empreendida ao longo desta pesquisa buscou contribuir para o preenchimento da enorme lacuna de conhecimento acerca da deposição/precipitação geoquímica nas salinas solares no Brasil. Nesse sentido, verifica-se que esses ambientes evaporíticos/hipersalinos apresentam uma dinâmica geoquímica no espaço e no tempo, onde o conhecimento existente sobre a diversidade e potencial uso dos recursos naturais aí encontradas aí são incipientes. Para tanto, o manejo integrado das salinas implica na necessidade de acompanhamento contínuo e realização de estudos mais aprofundados acerca da caracterização dos processos biogeoquímicos que ocorrem ao longo do circuito de produção de sal.

Portanto, esta abordagem dará suporte para as decisões sobre as práticas de gestão e manejo das áreas atualmente ocupadas pelas salinas, uma vez que estas são o único sistema biológico capaz de ser instalado em planícies hipersalinas sem a necessidade de intervenção geotécnica. É necessário frisar que a maior parte das salinas foram implantadas efetivamente nas áreas onde ocorria naturalmente o processo de deposição evaporítica natural de sais de origem marinha, através da evaporação solar nas planícies hipersalinas onde as águas das marés ficavam acumuladas. Em termos econômicos e de reduzido impacto ambiental, as salinas podem ser consideradas a única alternativa para a ocupação dessas planícies, uma vez que evaporação natural da água do mar implica na constante precipitação de sais (e.g. gipsita, halita, biscofita, silvita e epsomita) na superfície do solo, acarretando em um processo de salinização natural. Estes sais, em virtude do longo período de evaporação, ficam retidos no solo em forma de diversas camadas (de acordo com os ciclos evaporíticos), onde a inundação destas áreas apenas veio a proporcionar o controle/manejo da evaporação da salmoura, possibilitando a extração de uma maior quantidade de sal.

Espera-se que este trabalho possa vir a contribuir com uma nova avaliação sobre salinas brasileiras, de maneira que possam ser repensadas estratégias tanto para a implantação de novas salinas quanto para a efetiva definição de ações voltadas para a sua conservação. Nesse sentido, os diferentes aspectos da deposição geoquímica nas salinas brasileiras foram caracterizados, os quais 
poderão ser tomados como referenciais iniciais para novas investigações, assim como para serem inseridos nos sistemas de gestão.

\section{REFERÊNCIAS}

AL-FARRAJ, A. An evolutionary model for sabkha development on the north coast of the UAE. Journal of Arid Environments, v. 63, n. 4, p.740-755, 2005.

BAAS-BECKING, L. G. M. Historical notes on salt and salt-manufacture. Scient Mon., v. 32, p. 434-446, 1931.

BASEgGiO G. The Composition of seawater and its concentrates. In Proceedings of the Fourth Symposium on Salt, 8 - 12 April 1973. Houston: Edited by Alan H. Coogan: Open Library, 1973. 351-358 p.

BAUDIN, J. P. Contribution a l'étude écologique dês milieux saumatres méditerranéens. Vie et Milieu, v. 30, p. 121-129, 1980.

BRITTON, R. H.; JOHNSON, A. R. An ecological account of a Mediterranean salina: the Salin de Giraud, Camargue (S. France). Biol Conserv., v. 42, p.185-230, 1987.

COLEMAN, M. J. Carbon sequestration in benthic mats of solar ponds. In Proceedings of the 9th International Symposium on Salt. Beijing: Sha Zuoliang (Ed.), Gold Wall Press, Vol. A: 2009. 765-775 p.

COPELAND, B. J. Environmental characteristics of hypersaline lagoons. Texas University Contributions to Marine Science, v. 12, p. 207-218, 1967.

COSTA, D. F. S.; DE MEDEIROS ROCHA, R.; CÂNDIDO, G. A. Perfil de sustentabilidade e uso dos recursos naturais em salinas solares no estuário do Rio Apodi-Mossoró (RN). In Desenvolvimento Sustentável e Sistemas de Indicadores de Sustentabilidade: formas de aplicações em contextos geográficos diversos e contingências específicas. Campina Grande - PB: Editora da Universidade Federal de Campina Grande, 2010. 401 - 426 p.

COSTA, D. F. S. Caracterização ecológica e serviços ambientais prestados por salinas tropicais. Tese de Doutorado apresentado no programa de Pós-graduação em Biologia / Departamento de Biologia, Universidade de Aveiro, Aveiro - Portugal, 2013. 193 p.

COSTA, D. F. S.; DE MEDEIROS ROCHA, R.; LUCENA FILHO, M. A.; SILVA, A. A. Breve revisão sobre a evolução histórica da atividade salineira no Estado do Rio Grande do Norte (Brasil). Sociedade \& Natureza, v. 25, n. 1, p. 21-34, 2013.

CURTIS, R.; EVANS, G.; KINSMAN, D. J. J.; SHEARMAN, D. J. Association of dolomite and anhydrite in the recent sediments of the Persian Gulf. Nature, v. 197, p. 6779-6800, 1963.

DAVIS, J. Structure, function, and management of the biological system for seasonal solar saltworks. Glob. Nest J., v. 2, n.3, p.217-226, 2000. 
DERBY, I. H.; VICTOR, I. The dissociation tensions of certain hydrated chlorides and the vapour pressure of their saturated solutions. I. Amer. Chem. Soc., v. 38, n. 8, p. 1439-1451, 1916.

EMPRESA BRASILEIRA DE PESQUISA AGROPECUÁRIA - EMBRAPA. Sistema brasileiro de classificação de solos. 2 ed. Brasília/DF: Centro Nacional de Pesquisas de Solos, 1999. 306 p.

EVAGElOPOUlOS, A.; KOUTSOUBAS, D.; BASSET, A.; PINNA, M.; DIMITRIADIS, C.; SANGIORGIO, F.; BARBONE, E.; MAIDANOU, M.; KOULOURI, P.; DOUNAS, C. Spatial and seasonal variability of the macrobenthic fauna in Mediterranean solar saltworks ecosystems. Aquatic Conserv: Mar. Freshw. Ecosyst., v. 18 p.118-134, 2008.

EVANS, G. Coastal and nearshore sedimentation: a comparison of clastic and carbonate deposition. Proceedings of Geological Society of London, v. 81, p. 493-508, 1970.

FAO - Food and Agriculture Organization of ONU. World Reference Base for Soil Resources. World Soil Resources Report 103. Rome: FAO, 2006. 132 p.

FERNÂNDEZ-LOZANO, J. K. Recovery of epsomite and sylvite from seawater bittern by crystallization. In Proceedings of the Fourth Symposium on Salt, 8 - 12 April. Houston: Edited by Alan H. Coogan: Open Library; 1973. 501-510 p.

GIORDANO, M.; BEARDALL J. Impact of environmental conditions on photosynthesis, growth and carbon allocation strategies of hypersaline species of Dunaliella. Global NEST Journal, v. 11, n.1, p.79-85, 2009.

GUO, G; ARAYA, K; JIA, H; ZHANG, Z; OHOMIYA, K; MATSUDA, J. Improvement of saltaffected soils, part 1: interception of capillarity. Biosystems Engineering, v. 94, n. 1, p. 139-150, 2006.

HUAI-KE, W.; GUAN-QIANG, J. The Utilization and environmental protection of Qinghai Salt Lake Resource, In Proceedings of the 9th International Symposium on Salt. Beijing: Sha Zuoliang (Ed.), Gold Wall Press, Vol. B. 2009. 913-916 p.

JADHAV, M. H. Recovery of Crystalline magnesium chloride-hexahydrate by solar evaporation of sea bitterns. In Proceedings of the Sixth International Symposium on Salt, May 24-28 May 1983; Toronto: Edited by Schreiber BC and Harner HL: Salt Institute, 1983, 417-449 p.

JAVOR, B. J. Geology and Chemistry. In Hypersaline environments - microbiology and biogeochemistry. Berlin: Spinger-Verlag, 1989. 5-25 p.

KENDALL, A. C. Evaporites. In Facies Models. 2 ed. Toronto: Geoscience Canada Reprint Series 1, 1984. 259-296 p.

KENDALL, A. C.; HARWOOD, G. M. Marine evaporites: arid shorelines and basins. In Sedimentary environments: processes, facies and stratigraphy. 3 ed. Oxford: Blackwell, 1996. 281-324 p.

KHEMAKHEM, H.; ELLOUMI, J.; MOUSSA, M.; ALEYA, L.; AYADI, H. The concept of ecological succession applied to phytoplankton over four consecutive years in five ponds featuring a salinity gradient. Estuarine, Coastal and Shelf Science, v.88, p. 33-44, 2010. 
KOROVESSIS N. A., LEKKAS T. D. Solar saltwork's wetland function. Global NEST Journal, v. 11, n. 1, p. 49-57, 2009.

LÓPEZ, E.; AGUILERA, P. A.; SCHMITZ, M. F.; CASTRO, H.; PINEDA, F. D. Selection of ecological indicators for the conservation, management and monitoring of Mediterranean coastal salinas. Environ. Monit. Assess., v. 166 n. 3, p. 241-256, 2010.

MARGALEF, R. Ecología. Barcelona: Omega. 1974.

MElO, P. R. C.; CARVAlho, R. S.; PINTO, D. C. Halita. In CETEM. Rochas e Minerais Industriais Rochas Minerais Industriais: usos e especificações. Rio de Janeiro: CETEM/MCT, 2008. 551- $584 \mathrm{p}$.

MENDES, A. M. S.; FONTES, R. L. F.; OLIVEIRA, M. Variabilidade espacial da textura de dois solos do Deserto Salino, no Estado do Rio Grande do Norte. Rev. Ciên. Agron., v. 39, n. 1, p. 19$27,2008$.

MIRANDA, L. B.; CASTRO, B. M.; KJERFVE, B. Princípios de oceanografia física de estuários. São Paulo: Editora da Universidade de São Paulo, 2002.

MOREIRA, E. G. S.; DEMATTÊ, J. L. I.; MARCONI, A. Caracterização e gênese de solonetz solodizado do sertão central e do médio Jaguaribe - Estado do Ceará. An. ESALQ., v. 46, n. 2, p. 319-341, 1989.

OREN, A. Solar salterns. In Halophilic microorganisms and their environments: cellular origin Life in extreme habitats and astrobiology. New York: Kluwer Academic Publishers, 2002. 441-469 p.

OREN, A. Saltern evaporation ponds as model systems for the study of primary production processes under hypersaline conditions. Aquat. Microb. Ecol., v. 56, p. 193-204, 2009.

ORTIZ-MILAN, S. M. Project of recovery the biological conditions of the production system in saltworks of Industria Salinera de Yucatan S.A. de C.V. (ISYSA) Damaged by the hurricane isidore in september of 2002. Global NEST Journal, v. 11, n. 1, p. 91-95, 2009.

PEDRÓS-ALIÓ, C.; CALDERÓN-PAZ, J. I.; MACLEAN, M. H.; MEDINA, G.; MARRASÉ, C.; GASOL, J. M; GUIXA-BOIXEREU, N. The microbial food web along salinity gradients. FEMS Microbiol Ecol., v. 32, p. 143-155, 2000.

RAHAMAN, A. A.; VENKAT, R.; PATEL, D. K.; PATEL, K. K. Environmental impact assessment of crystalline saltworks, Gongoni Malindi district, Kenya. In Proceedings of the 9th International Symposium on Salt. Beijing: Sha Zuoliang (Ed.), Gold Wall Press, Vol. B. 2009. $1122-1128 \mathrm{p}$.

SILVA, M. M.; SCHREIBER, B. C.; SANTOS, C. L. Evaporites as mineral resources. Rev. Bras. Geof., v. 18, n. 3, p. 338-350. São Paulo: 2000.

SILVA, A. M. A.; BARBOSA, J. E. L.; MEDEIROS, P. R.; ROCHA, R. M.; LUCENA FILHO, M. A.; COSTA, D. F. S. Zooplankton (Cladocera and Rotifera) variations along a horizontal salinity 
gradient and during two seasons (dry and rainy) in a tropical inverse estuary (Northeast Brazil). Pan-American Journal of Aquatic Sciences, v. 4, n. 2, p. 226-238, 2009.

SOUTO, F. J. B.; WATANABE, T.; ROCHA, R. M.; BARBOSA, J. E. L. Distribuição espaçotemporal da comunidade fitoplanctônica e variáveis hidrológicas em uma salina artesanal do estado do Rio Grande do Norte, Brasil. Revista Nordestina de Biologia, v. 15, n. 2, p. 7-26. João Pessoa: 2000.

TAKEKAWA, J. Y.; MILES, A. K.; SCHOELLHAMER D. H. Trophic structure and avian communities across a salinity gradient in evaporation ponds of the San Francisco Bay estuary. Hydrobiologia, v. 567, p. 307-327, 2006.

VITA, S.; LUNA, F. J.; TEIXEIRA, S. Descrições de técnicas da química na produção de bens de acordo com os relatos dos naturalistas viajantes no Brasil colonial e imperial. Quim. Nova, v. 30, n.5, p. 1381-1386. São Paulo: 2007.

\section{AGRADECIMENTOS}

A equipe agradece ao Laboratório de Biogeografia e ao Centro de Ensino Superior do Seridó (UFRN - Campus de Caicó), pelo apoio nos trabalhos de campo e de gabinete, assim como a PROPESQ/UFRN (PVF10463-2014) e ao CNPq (MCTI/CNPQ/Universal Proc.447227/2014-9), pelo apoio financeiro.

Recebido em: 06/04/2018

Aceito para publicação em: 28/06/2018 